\title{
Multiplicative cross-correlated noise induced escape rate from a metastable state
}

\author{
Jyotipratim Ray Chaudhuri, ${ }^{1, *}$ Sudip Chattopadhyay, ${ }^{2,0}$ and Suman Kumar Banik ${ }^{3, \text { 团 }}$ \\ ${ }^{1}$ Department of Physics, Katwa College, Katwa, Burdwan 713130, India \\ ${ }^{2}$ Department of Chemistry, Bengal Engineering and Science University, Shibpur, Howrah 711103, India \\ ${ }^{3}$ Department of Physics, Virginia Polytechnic Institute and State University, Blacksburg, VA 24061-0435, USA
}

(Dated: October 30, 2018)

\begin{abstract}
We present an analytical framework to study the escape rate from a metastable state under the influence of two external multiplicative cross-correlated noise processes. Starting from a phenomenological stationary Langevin description with multiplicative noise processes, we have investigated the Kramers' theory for activated rate processes in a nonequilibrium open system (one-dimensional in nature) driven by two external cross-correlated noise processes which are Gaussian, stationary and delta correlated. Based on the Fokker-Planck description in phase space, we then derive the escape rate from a metastable state in the moderate to large friction limit to study the effect of degree of correlation on the same. By employing numerical simulation in the presence of external crosscorrelated additive and multiplicative noises we check the validity of our analytical formalism for constant dissipation, which shows a satisfactory agreement between both the approaches for the specific choice of noise processes. It is evident both from analytical development and the corresponding numerical simulation that the enhancement of rate is possible by increasing the degree of correlation of the external fluctuations.
\end{abstract}

PACS numbers: 05.40.-a, 02.50.Ey, 82.20.Uv

\section{INTRODUCTION}

Rate theory deals with the passage of a system from one stable local minima of the energy landscape to another via a potential energy barrier providing relevant information on the long-time behavior of the system with different metastable states and hence is a very useful and pedagogical model for the microscopic description and understanding of a wide range of physical, chemical and biological phenomena. Examples include diffusion of atoms in solids or on surfaces, isomerization reactions in solution, electron transfer processes, and ligand binding in proteins or protein folding reactions. Not only that the phenomena of flux transitions in superconducting quantum interference devices can also be modeled by rate theory. Kramers 1 pointed out that the passage of a system from a metastable state due to thermal agitation can be considered as a model Brownian particle trapped in a one-dimensional well representing the reactant state which is separated by a barrier of finite height from a deeper well signifying the product state. The particle is supposed to be immersed in a medium such that the medium exerts damping force on the particle but at the same time thermally activates it so that the particle may gain enough energy from its surroundings to cross the barrier. Thus, the surrounding fluid provides friction and random noise. Conventionally, the friction is assumed to be Markovian and the noise, Gaussian and white. Kramers theory provides a useful framework for the explanation and prediction of rates and mechanisms for various barrier crossing phenomena and for chemical reactions in particular $2,3,4,5,6,7,8,9,10$

Most of the above mentioned works are basically dealt either with phenomenological Langevin equation or by microscopic system-reservoir formulation with both bi- linear or non-linear coupling scheme. Although, the effect of system-bath linear coupling and associate consequences in different branches of molecular sciences is well studied, the modeling and the proper microscopic interpretation of the nature of non-linear coupling and corresponding manifestation on the various molecular phenomena is a very challenging task posed in front of researchers in this field. Tanimura and co-workers 11 explained the phenomena of elastic and inelastic relaxation mechanism and their cross effect in vibrational and Raman spectroscopy using non-linear system-bath model.

It is now a well documented fact that solvent plays a very crucial role in computing the dynamics of a Brownian particle $12,13,14,15,16,17,18,19,20$ The dynamics of a model Brownian particle in an inhomogeneous solvent generally leads to state dependent diffusion due to the appearance of multiplicative noise and state dependent dissipation $19,20,21$ Although modeling of Brownian motion in presence of inhomogeneous solvent is a nontrivial task, several approaches have been made to study different aspects of molecular sciences, e.g., activated rate processes, $, 5,21,22,23$ noise induced transport, $19,20,24,25,26$ stochastic resonance $\frac{27}{7}$ and laser and optics $\underline{28}$

In the overwhelming majority of the above mentioned treatments, fluctuations experienced by the system of interest is of internal origin so that the fluctuations and dissipation get related through the celebrated fluctuation-dissipation relation (FDR) $\stackrel{29}{2}$ However, in some situations, the physical origin of dissipation and of fluctuations are different as well as independent. $19,20,21,30,31,32,33,34,35$ Thus, there exists no balance between the influx (efflux) of energy to (from) the system and hence there exist no FDR. As a result of this, such systems (usually termed as nonequilibrium open system $^{36}$ ) do not approach thermal equilibrium asymp- 
totically and many interesting phenomena are observed thereof 30,31

To the best of our knowledge, creation of an open system in a nonequilibrium process can be realized in many ways. To mention, we briefly discuss about few of them in the following which are relevant to the present work. An additional perturbation applied to the heat bath can excite few bath modes which effectively leads to the creation of a nonstationary process where the nonstationarity mainly gets reflected in the dissipation kernel.13,14,17,37 Dynamics in this case is mainly guided by an irreversible process. ${ }^{14,17}$ An extensive analysis of this approach can be found in the very recent work of Popov and Hernandez. ${ }^{17}$ External perturbation on the other hand can directly excite the system mode itself (leaving the bath mode alone) or the local bath modes (leaving the system mode alone). In both the situations the additional energy input by an independent source leads to a shift in the temperature, thus creating an effective temperature like quantity, 19,20,21,32,33,34,38 keeping the underlying dynamical process stationary. Another immediate effect of the breakdown of FDR in this case is the creation of a steady state instead of an equilibrium state in the long time limit. To study the escape rate from a metastable state under the influence of external cross-correlated noise processes we have adopted one of the situations mentioned in the latter case to create an open system. In the present work we drive the reaction coordinate (the system mode) by multiplicative cross-correlated noise processes, leaving the bath modes as it is. In such a situation, the Brownian particle is energized by an extra input of energy, in addition to the thermal energy provided by the heat bath. This leads to, what we have tried to study in this paper, an enhancement of barrier crossing event in the activated rate processes within the framework of Markovian stationary dynamics.

The barrier crossing dynamics with multiplicative and additive white noise processes aroused strong interest in the early eighties, where noise forces that are present simultaneously in the dynamical system were usually treated as random variables uncorrelated with each other. However, there are situations where fluctuations in some stochastic process may have common origin. If this happens then the statistical properties of the fluctuations should not be very much different and can be correlated to each other. Nonlinear stochastic systems including noise terms have drawn interest on a wide scale owing to their numerous applications in the field of molecular sciences. Generally it is observed that under such a situation, the noise affects the dynamics through a system variable. The cross-correlated noise processes were first considered by Fedchenia 39 in the context of hydrodynamics of vortex flow where the author introduced crosscorrelation among the fluctuations from a common origin that appear in the time evolution equation of dimensionless modes of flow rates. The interference of additive and multiplicative white noise processes in the kinetics of the bistable systems was first considered by Fulinski and Telejko 40 where they mentioned the physical possibility of a cross-correlated noise. Madureira et al. $\stackrel{41}{n}$ have pointed out the probability of cross-correlated noise in a realistic model (ballast resistor) showing bi-stable behavior of the system. Recently, Mei et $a l^{42}$ have studied the effects of correlations between additive and multiplicative noise on relaxation time in a bi-stable system driven by cross-correlated noise.

It is now well accepted that the effect of correlation between additive and multiplicative noise (which is also termed as correlated noise processes) is considered indispensable in explaining phenomena such as steady state properties of a single mode laser, 43 bistable kinetics,, 44 stochastic resonance in linear system, $\stackrel{45}{,}$ steady-state entropy production, $\stackrel{46}{ }$ stochastic resonance $\frac{47}{}$ and transport of particles, $\stackrel{48}{ }$ etc. Zhu ${ }^{43}$ investigated theoretically the statistical fluctuations of a single mode laser that include correlations between additive and multiplicative white noises and showed that the effect of correlation can lead to larger intensity fluctuations. One can utilize this intensity fluctuations to induce a narrow peak in the probe absorption spectrum as well as significantly modify the emission spectra of matter strongly resonant with laser field. As shown by $\mathrm{Ai}$ et al. $\frac{49}{}$ one can use the logistic differential equation to analyze the effects of environmental fluctuations on cancer cell growth using correlated Gaussian white noise scheme whereby the degree of correlation of the noise (environmental intensive fluctuations) can cause tumor cell extinction. Berdichevsky and Gitterman 45 showed that the maxima of signal to noise ratio, as a function of the asymmetry of noise, disappears in the absence of the coupling between additive and multiplicative white noises. Very recently Ghosh et $a l .50$ have used multiplicative correlated noise processes to study the splitting of Kramers' rate in a symmetric triple well potential.

As the presence of the cross-correlated noise changes the Langevin dynamics of the system, 38,51 it is expected that there may exist some additional effect of crosscorrelation on the escape rate of a metastable state. Study of this additional effect can perfectly serve as a motivation of our work presented in this paper. To achieve this we extend our recently developed theoretical approach 38 to study the effect of multiplicative crosscorrelated noise on the escape rate from a metastable state. In this present study, the external fluctuation applied to the system under consideration is assumed to be independent of the system's characteristic dissipation. In this article, we study the reaction rate (in one dimension) under the influence of both the internal (thermal) noise and external (non-thermal) cross-correlated noises simultaneously to examine the role of correlation between external delta correlated fluctuations on the escape rate from a metastable state when the dissipation is space dependent and the noises appear multiplicatively in the dynamical equation. In our model the system is externally driven by two cross-correlated fluctuations. 
Starting from phenomenological Langevin equation with space dependent dissipation and multiplicative noises, we construct the corresponding Fokker-Planck equation in phase space. Under proper boundary conditions, we solve the Fokker-Planck equation to calculate the escape rate for moderate to large dissipation. In the numerical implementation of our development the steady state rate expression (derived analytically) is checked with stochastic simulation to establish the fact that the incorporation of external perturbation through cross-correlated fluctuations in to the traditional Kramers' model enhances the rate across the barrier top.

The organization of the paper is as follows. In section II, starting from a phenomenological Langevin equation for an open system nonlinearly coupled with the environment and simultaneously acted upon by two crosscorrelated multiplicative white noise processes, we construct the Fokker-Planck description of the underlying stochastic process which is multiplicative in general. We then calculate the escape rate from a metastable state to examine the barrier crossing dynamics. A typical example has been considered in section III to study the effect of the cross-correlated fluctuations on the escape rate. The paper is then concluded in section IV. To make the paper self contained we provide the derivation of the FokkerPlanck equation and the calculation of escape rate in the two appendices.

\section{CORRELATED NOISE INDUCED ESCAPE FROM A METASTABLE STATE}

We consider the motion of a particle of unit mass moving in a Kramers type potential $V(x)$ such that it is acted upon by random force $f(t)$ of internal origin, i.e., $f(t)$ originates due to the coupling of the system with its environment and hence is connected to the friction through the fluctuation-dissipation relation. Apart from the internal noise, we assume that the system is acted upon by two external Gaussian noise processes, $\epsilon(t)$ and $\pi(t)$, both of which have a common origin and consequently are correlated. Thus, from the very mode of description of our model, it is evident that the system is open in nature as it is driven externally by two correlated fluctuations. The dynamics of the particle is governed by the Langevin equation

$\ddot{x}=-\Gamma(x) \dot{x}-V^{\prime}(x)+h(x) f(t)+g_{1}(x) \epsilon(t)+g_{2}(x) \pi(t)$,

with

$$
h(x)=\sqrt{k_{B} T \Gamma(x)},
$$

where $\Gamma(x)$ is the space dependent friction that arises due to non-linear system-reservoir coupling. ${ }^{36}$ Here, $g_{1}(x)$ and $g_{2}(x)$ are two arbitrary smooth functions of $x$ and their presence makes the external noise processes multiplicative. $T$ is the thermal equilibrium temperature and
$k_{B}$ is the Boltzmann constant. $\epsilon(t)$ and $\pi(t)$ are Gaussian white noise processes with statistical properties

$$
\begin{aligned}
\langle\epsilon(t)\rangle & =\langle\pi(t)\rangle=0 \\
\left\langle\epsilon(t) \epsilon\left(t^{\prime}\right)\right\rangle & =2 D_{\epsilon} \delta\left(t-t^{\prime}\right) \\
\left\langle\pi(t) \pi\left(t^{\prime}\right)\right\rangle & =2 D_{\pi} \delta\left(t-t^{\prime}\right) \\
\left\langle\epsilon(t) \pi\left(t^{\prime}\right)\right\rangle & =\left\langle\pi(t) \epsilon\left(t^{\prime}\right)\right\rangle=2 \lambda \sqrt{D_{\epsilon} D_{\pi}} \delta\left(t-t^{\prime}\right)
\end{aligned}
$$

In the above equations (3b] $3 \mathrm{~d}), D_{\epsilon}$ and $D_{\pi}$ are the strength of the fluctuations $\epsilon(t)$ and $\pi(t)$, respectively and $\lambda(0 \leqslant \lambda<1)$ denotes the degree of correlation between the noise processes $\epsilon(t)$ and $\pi(t)$. The internal noise $f(t)$ is also assumed to be Gaussian and delta correlated with statistical properties

$$
\langle f(t)\rangle=0 \text { and }\left\langle f(t) f\left(t^{\prime}\right)\right\rangle=2 \delta\left(t-t^{\prime}\right) .
$$

In the above equations $\langle\cdots\rangle$ implies the average over the realizations of the noise (external or internal) processes. Eq.(2) along with the second relation of Eq.(4) comprises the fluctuation-dissipation which relates the damping function $\Gamma(x)$ with the fluctuation $f(t)$. The external noise processes $\epsilon(t)$ and $\pi(t)$ are independent of the dissipation function and there is no corresponding fluctuation-dissipation relation. We further assume that $f(t)$ is independent of $\epsilon(t)$ and $\pi(t)$ so that

$$
\left\langle f(t) \epsilon\left(t^{\prime}\right)\right\rangle=\left\langle f(t) \pi\left(t^{\prime}\right)\right\rangle=0 .
$$

In the absence of the external noise processes, the system being closed, the fluctuation-dissipation relation eventually brings it to a stationary state and consequently one can examine the barrier dynamics of the system using standard methods applicable for a thermodynamically closed system. The external fluctuations and their correlation modify the dynamics of activation in two ways. First, they influence the dynamics in the region around the barrier top so that the effective stationary flux across it gets modified. Second, in the presence of these fluctuations, the equilibrium distribution of the source well is disturbed so that one has to consider a new stationary distribution, if any, instead of the standard equilibrium Boltzmann distribution. This new stationary distribution must be a solution of the FokkerPlanck equation around the bottom of the source well region and serves as an appropriate boundary condition analogous to Kramers problem.

Keeping this in mind we write the corresponding Fokker-Planck equation in the phase space [i.e. in $(x, v)$ space of the system, where $v=\dot{x} \equiv d x / d t]$, describing the dynamics of the Langevin equation (11) [see Appendix-A for detailed derivation]

$$
\begin{aligned}
\frac{\partial P}{\partial t}= & -v \frac{\partial P}{\partial x}+\left[\Gamma(x) v+V^{\prime}(x)\right] \frac{\partial P}{\partial v}+A(x) \frac{\partial^{2} P}{\partial v^{2}} \\
& +\Gamma(x) P,
\end{aligned}
$$


where

$$
\begin{aligned}
A(x)= & k_{B} T \Gamma(x)+g^{2}(x) \\
g(x)= & \left\{D_{\epsilon} g_{1}^{2}(x)+2 \lambda \sqrt{D_{\epsilon} D_{\pi}} g_{1}(x) g_{2}(x)\right. \\
& \left.+D_{\pi} g_{2}^{2}(x)\right\}^{1 / 2} .
\end{aligned}
$$

The diffusion coefficient $A(x)$ in the Fokker-Planck equation (6) is an implicit function of the correlation parameter $\lambda$ (see the expression of $g(x)$ ). Thus by increasing the value of $\lambda$ one can increase the value of the diffusion coefficient. This property gets directly reflected in the final rate expression and in the expression of effective temperature (see Eqs.(12) and (13)). In deriving Eq.(6), we have made use of Eqs.(3a) $3 \mathrm{~d}$ ) and (4) and the fact that $f(t)$ is independent of $\epsilon(t)$ and $\pi(t)$. It should be noted that when the noise is purely internal and for linear system-reservoir coupling, Eq.(6) reduces to the Kramers equation. 1

Kramers' model for a chemical reaction consists of a particle undergoing Brownian motion whose coordinate $x$ corresponds to the reaction coordinate and $v=d x / d t$ the reaction rate. In addition to that in Kramers' original treatment, $\frac{1}{-}$ the dynamics of the Brownian particle was governed by Markovian random process. Since the work of Kramers, a number of workers have extended Kramers model for non-Markovian case and for state dependent diffusion to derive the expression for the escape rate.$\underline{\underline{5}} \mathrm{In}$ order to allow ourselves a comparison with the FokkerPlanck equation of other forms, we note that though the underlying dynamics is Markovian, the diffusion coefficient in Eq. (8) is coordinate dependent. It is customary to get rid of this dependence by approximating the coefficients at the barrier top or potential well where we need the steady-state solution of Eq.(8). One may also use mean field solution of Eq.(8) obtained by neglecting the fluctuation terms and putting approximate stationary condition in the diffusion coefficient.

For harmonic oscillator with frequency $\omega_{0}, V(x) \approx$ $E_{0}+\omega_{0}^{2}\left(x-x_{0}\right)^{2} / 2$, the linearized version of the FokkerPlanck equation can be represented as

$$
\begin{aligned}
\frac{\partial P}{\partial t}= & -v \frac{\partial P}{\partial x}+\Gamma\left(x_{0}\right) P+\left[\Gamma\left(x_{0}\right) v+\omega_{0}^{2}\left(x-x_{0}\right)\right] \frac{\partial P}{\partial v} \\
& +A_{0} \frac{\partial^{2} P}{\partial v^{2}}
\end{aligned}
$$

where $A_{0}=k_{B} T \Gamma\left(x_{0}\right)+g^{2}\left(x_{0}\right)$, is calculated at the bottom of the potential $\left(x \approx x_{0}\right)$. The general steady state solution of Eq.(9) becomes

$$
P_{0}^{s t}(x, v)=\frac{1}{Z} \exp \left(-\frac{v^{2}}{2 D_{0}}-\frac{\omega_{0}^{2}\left(x-x_{0}\right)^{2}}{2 D_{0}}\right),
$$

with $D_{0}=A_{0} / \Gamma\left(x_{0}\right)$ and $Z$ is the normalization constant. The solution (10) can be verified by direct substitution in the steady state version of the Fokker-Planck equation (9), namely

$$
\begin{aligned}
& -v \frac{\partial P_{0}^{s t}}{\partial x}+\Gamma\left(x_{0}\right) P_{0}^{s t}+\left[\Gamma\left(x_{0}\right) v+\omega_{0}^{2}\left(x-x_{0}\right)\right] \frac{\partial P_{0}^{s t}}{\partial v} \\
& +A_{0} \frac{\partial^{2} P_{0}^{s t}}{\partial v^{2}}=0 .
\end{aligned}
$$

It should be noted that the distribution (10) is not an usual equilibrium distribution, rather it serves as stationary distribution for the non-equilibrium open system. This stationary distribution plays the role of an equilibrium distribution of the closed system which can, however, be recovered in the absence of the external noise.

We now embark on the problem of decay of a meta stable state in the presence of external cross-correlated noise processes. In Kramers' approach, the particle coordinate $x$ corresponds to the reaction coordinate, and its values at the minima of the potential well $V(x)$ are separated by a potential barrier to describe the reactant and product states. Linearizing the motion around the barrier top at $x \approx x_{b}$, the steady state version of the Fokker-Planck equation corresponding to Eq.(6) reads as

$$
\begin{aligned}
& -v \frac{\partial P_{b}^{s t}}{\partial x}-\omega_{b}^{2}\left(x-x_{b}\right) \frac{\partial P_{b}^{s t}}{\partial v}+\Gamma\left(x_{b}\right) \frac{\partial\left(v P_{b}^{s t}\right)}{\partial v} \\
& +A_{b} \frac{\partial^{2} P_{b}^{s t}}{\partial v^{2}}=0,
\end{aligned}
$$

where, $V(x) \approx E_{b}-\omega_{b}^{2}\left(x-x_{b}\right)^{2} / 2$ with $\omega_{b}^{2}>0$, and the suffix ' $b$ ' indicates that coefficients are to be calculated using the general definition of $A$ at the barrier top.

After imposing appropriate physically motivated boundary conditions we then derive the escape rate for nonequilibrium open systems valid in the moderate to strong friction limit [see Appendix-B for detailed calculation]

$$
k=\frac{\omega_{0}}{2 \pi} \frac{D_{b}}{\sqrt{D_{0}}} \sqrt{\frac{\Lambda}{1+\Lambda D_{b}}} \exp \left(-\frac{E}{D_{b}}\right),
$$

where $E=E_{b}-E_{0}$ is the potential barrier height, and $D_{b}=A_{b} / \Gamma\left(x_{b}\right)$. Already we have mentioned in the introduction, open system mean the system is not thermodynamically closed as it is driven by two noises of external origin and consequently there is no fluctuationdissipation relation which is mainly responsible to establish the thermal equilibrium (for closed system). It is also important to note that the escape from a metastable state intrinsically a non-equilibrium phenomenon as fluctuations establish a steady mass motion to give rise to a non-vanishing constant current over the potential barrier. In the steady state rate expression (12), all information about the thermal temperature due to internal noise processes, the strength of both the external noises and the position dependent dissipation, $\Gamma(x)$, are hidden in the quantities $D_{0}, D_{b}$ and $\Lambda$. Furthermore, the rate constant $k$ is an implicit function of the degree of correlation, $\lambda$, between the external noise processes. We conclude this section by mentioning the fact that the $\lambda$-dependence 
of the effective temperature $\left(D_{b}\right)$ of our model does not depend on the detailed forms of the coupling functions $g_{1}(x)$ and $g_{2}(x)$.

\section{ANALYSIS OF THE INTERFERENCE BETWEEN TWO EXTERNAL FLUCTUATIONS}

In this section we present the numerical implementation of our presently developed theory to establish its potentiality and applicability to demonstrate the effect of correlation of two external noises $\epsilon(t)$ and $\pi(t)$ on the rate. Eq. (12) is the theoretical expression for the escape rate and is applicable for any two functions $g_{1}(x)$ and $g_{2}(x)$ appeared in equation (11). To test the validity of the rate expression (12), one needs to assume the particular forms of $g_{1}(x)$ and $g_{2}(x)$. In addition to that an explicit form of the damping term $\Gamma(x)$ is needed. For simplicity we use a constant dissipation $\Gamma(x)=\gamma$ in our numerical simulation. Thus the explicit expression for the escape rate for constant dissipation and arbitrary $g_{1}(x)$ and $g_{2}(x)$ becomes

$$
\begin{aligned}
k= & \frac{\omega_{0}}{2 \pi \omega_{b}}\left[\frac{\gamma k_{B} T+g^{2}\left(x_{b}\right)}{\gamma k_{B} T+g^{2}\left(x_{0}\right)}\right]^{1 / 2}\left[\left(\frac{\gamma^{2}}{2}+\omega_{b}^{2}\right)^{1 / 2}-\frac{\gamma}{2}\right] \\
& \times \exp \left(-\frac{\gamma E}{\gamma k_{B} T+g^{2}\left(x_{b}\right)}\right)
\end{aligned}
$$

where the quantities $g\left(x_{0}\right)$ and $g\left(x_{b}\right)$ are evaluated around the potential minima $\left(x \approx x_{0}\right)$ and maxima $\left(x \approx x_{b}\right)$, respectively, using the function $g(x)$ defined in Eq.(8). In the above equation the quantity $g^{2}\left(x_{b}\right) / \gamma$ in the exponential factor together with the thermal energy $k_{B} T$ constitutes the effective temperature, a typical signature of the open system. $17,21,32,33,34,38$ Another effect of space dependent diffusion in the dynamics is the presence of reaction coordinate $\left(x_{0}\right.$ and $\left.x_{b}\right)$ in the steady state rate expression (13) which is typically absent in the standard Kramers' expression $\frac{1}{}$ (see Eq.(14) below). For $D_{\epsilon}=0$ and $D_{\pi}=0$, i.e., in the absence of external driving, Eq. (13) yields Kramers' rate expression for pure thermal fluctuations valid in the moderate to strong damping limit $\underline{1}$

$$
\begin{aligned}
k_{\text {Kramers }}= & \frac{\omega_{0}}{2 \pi \omega_{b}}\left[\left(\frac{\gamma}{2}^{2}+\omega_{b}^{2}\right)^{1 / 2}-\frac{\gamma}{2}\right] \\
& \times \exp \left(-\frac{E}{k_{B} T}\right),
\end{aligned}
$$

which can be also verified easily using the explicit forms of the parameters $D_{0}, D_{b}$ and $\Lambda$ in Eq.(12) in the limit $\epsilon(t)=\pi(t)=0$. It is thus clear that in the absence of the external noise processes, the derived rate expression (13) reduces to standard Kramers' escape rate in the moderate to large damping regime.

To study the dynamics, we consider a model cubic potential of the form $V(x)=b_{1} x^{2}-b_{2} x^{3}$ where, $b_{1}$ and

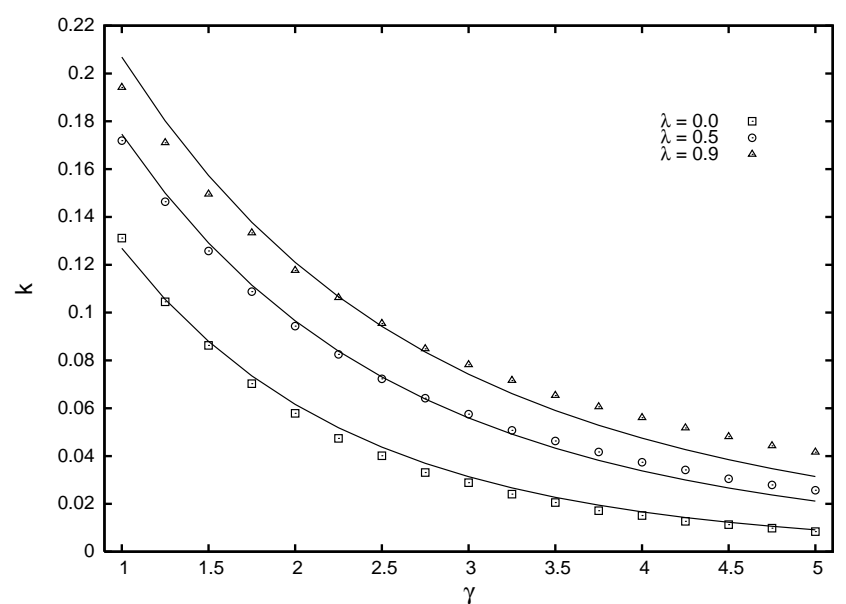

FIG. 1: Plot of barrier crossing rate $k$ as a function of dissipation constant $\gamma$ for various values of correlation parameter $\lambda$ and for $g_{1}(x)=x$ and $g_{2}(x)=1$. The solid lines are drawn from the theoretical expression, Eq. 113) and the symbols are the results of numerical simulation of Eq.(1). The values of the parameters used are $k_{B} T=0.1, D_{\epsilon}=D_{\pi}=0.1$ and $\lambda=$ $0,0.5$ and 0.9 .

$b_{2}$ are the two constant parameters with $b_{1}, b_{2}>0$, so that the potential barrier height becomes $4 b_{1}^{3} / 27 b_{2}^{2}$ and $x_{b}=2 b_{2} / 3 b_{1}$. In our numerical simulation we have used $b_{1}=b_{2}=1$. While numerically solving the Langevin equation (1) for constant dissipation, $\gamma$, we used a specific combination of the multiplicative terms, e.g., $g_{1}(x)=x$ and $g_{2}(x)=1$. Although both the external noise processes in our model are multiplicative in nature, the specific choice of the external noise processes we adopt in the numerical simulation captures the essential feature of our model (see the discussion in the following paragraph). We then numerically solve the Langevin equation (11) by employing stochastic simulation algorithm. ${ }^{53}$ The numerical rate has been defined as the inverse of the mean first passage time $21,32,34,54$ and has been calculated by averaging over 10,000 trajectories. To ensure the stability of our simulation, we have used a small integration time step $\Delta t=0.001$.

In Fig. 1, we show the comparison between the simulation result and theoretical expression where the escape rate $k$ is plotted as a function of the dissipation constant, $\gamma$, in the moderate to large damping regime where our theory is valid, for various values of the degree of correlation $\lambda$ and observe that for a given $\gamma$, the escape rate $k$ increases with an increase in $\lambda$. The result shows a reasonable agreement between the theory and the simulation. It is easy to check that for a particular combination of $g_{1}(x)$ and $g_{2}(x)$, by increasing the correlation parameter $\lambda$ one basically increases the value of the function $g(x)$ (see Eq.(8)) evaluated at $x \approx x_{b}$. This increment in $g\left(x_{b}\right)$ increases the effective temperature like quantity $k_{B} T+\left(g^{2}\left(x_{b}\right) / \gamma\right)$ for a fixed value of $\gamma$ which in turn pumps more energy into the system which eventually helps in crossing the barrier and increases the escape 
rate. Another way to explain this phenomenon is to look at the diffusion coefficient, $A_{b}$ across the barrier. From the expression of $A(x)$ (see Eq.(7)) evaluated at $x \approx x_{b}$, i.e., $A_{b}=\gamma k_{B} T+g^{2}\left(x_{b}\right)$ it is clear that $g\left(x_{b}\right)$ increases with an increase in $\lambda$ for a particular choice of $g_{1}(x)$ and $g_{2}(x)$ and for a fixed value of $\gamma k_{B} T$. This increment in the diffusion across the barrier enhances the reaction rate as observed. This is the central result of this paper.

\section{CONCLUSIONS}

In conclusion, we have extended our recently developed theoretical approach of studying escape rate from a metastable state under the influence of external additive cross-correlated noise processes 38 to investigate the effect of multiplicative noise. In contrast to our previous approach of using effective correlated noise constructed from two additive colored noise processes (see Eq.(1) of Ref. 38), the correlated noise used in the present work has been constructed from multiplicative white noises. The multiplicative nature of the correlated noises introduces a space dependent diffusion in the resultant Fokker-Planck equation (6) and modifies the exponential as well as the pre-exponential factor of the steady state rate expression (13). To check the validity of the steady state analytical rate expression, we have performed numerical simulation of the starting Langevin equation (1) with one multiplicative noise and the other additive one, which shows a satisfactory agreement between the theory and the numerics. The analytical development as well as the corresponding numerical simulation lead us to conclude that the enhancement of rate is possible by increasing the degree of correlation of the external fluctuations. So far, we have used the formalism of Markovian stochastic processes in this paper which can be extended to look into the dynamics within the framework of non-Markovian formalism. We plan to address this issue in our future communication.

\section{Acknowledgments}

JRC and SC would like to acknowledge the UGC, India for funding through the schemes PSW-103/06-07 (ERO) and 32-304/2006 (SR). SKB acknowledges support from Department of Physics, Virginia Tech.

\section{APPENDIX A: DERIVATION OF THE FOKKER-PLANCK EQUATION}

In this appendix we give the detailed calculation of constructing the Fokker-Planck equation (6) with space dependent diffusion corresponding to equation (1) which can be written as:

$$
\begin{aligned}
\dot{x} & =v, \\
\dot{v} & =-\Gamma(x) v-V^{\prime}(x)+h(x) f(t) \\
& +g_{1}(x) \epsilon(t)+g_{2}(x) \pi(t),
\end{aligned}
$$

We then rewrite the above equation in the following form:

$$
\begin{aligned}
& \dot{u_{1}}=F_{1}\left(u_{1}, u_{2}, t ; f(t), \epsilon(t), \pi(t)\right), \\
& \dot{u_{2}}=F_{2}\left(u_{1}, u_{2}, t ; f(t), \epsilon(t), \pi(t)\right),
\end{aligned}
$$

where we use the following abbreviations

$$
\begin{aligned}
u_{1}=x, u_{2}= & v, \\
F_{1}=v, F_{2}= & -\Gamma(x) v-V^{\prime}(x)+h(x) f(t)+g_{1}(x) \epsilon(t) \\
& +g_{2}(x) \pi(t) .
\end{aligned}
$$

The vector $u$ with components $u_{1}$ and $u_{2}$ thus represents a point in a two-dimensional 'phase space' and equation (A2) determines the velocity at each point in the phase space. The conservation of the phase points now asserts the following linear equations of motion for density $\rho(u, t)$ in phase space $e^{55}$

$$
\frac{\partial}{\partial t} \rho(u, t)=-\sum_{n=1}^{2} \frac{\partial}{\partial u_{n}} F_{n}(u, t, f(t), \epsilon(t), \pi(t)) \rho(u, t),
$$

or more compactly

$$
\frac{\partial \rho}{\partial t}=-\nabla \cdot F \rho .
$$

Our next task is to find out a differential equation whose average solution is given by $\langle\rho\rangle, \frac{55}{5}$ where the stochastic averaging has to be performed over both the internal noise process $f(t)$ and the external noise processes $\epsilon(t)$ and $\pi(t)$. To this end we note that $\nabla \cdot F$ can be partitioned into two parts: a constant part $\nabla \cdot F_{0}$ and a fluctuating part $\nabla \cdot F_{1}(t)$ containing these fluctuations. Thus, we write

$$
\begin{aligned}
& \nabla \cdot F(u, t, f(t), \epsilon(t), \pi(t)) \\
& =\nabla \cdot F_{0}(u)+\beta \nabla \cdot F_{1}(u, t, f(t), \epsilon(t), \pi(t)),
\end{aligned}
$$

where $\beta$ is a parameter (we put it as an external parameter to keep track of the perturbation equation; we put $\beta=1$ at the end of the calculation) and also note that $\left\langle F_{1}(t)\right\rangle=0$. Equation (A6) therefore takes the following form:

$$
\dot{\rho}(u, t)=\left(A_{0}+\beta A_{1}\right) \rho(u, t),
$$

where $A_{0}=-\nabla \cdot F_{0}$ and $A_{1}=\nabla \cdot F_{1}$. The symbol $\nabla$ is used for the operator that differentiates everything that comes after it with respect to $u$. Making use of one of the main results for the theory of linear equation of the form (A7) with multiplicative noise, 55 we derive an average 
equation for $\rho[\langle\rho\rangle=P(u, t)$ the probability density of $u(t)]$,

$$
\begin{aligned}
\frac{\partial P}{\partial t}= & \left\{A_{0}+\beta^{2} \int_{0}^{\infty} d \tau\left\langle A_{1}(t) \exp \left(\tau A_{0}\right) A_{1}(t-\tau)\right\rangle\right. \\
& \left.\times \exp \left(-\tau A_{0}\right)\right\} P .
\end{aligned}
$$

Equation (A8) is exact when the correlation times of fluctuations tend to zero. Using the expressions for $A_{0}$ and $A_{1}$ we obtain

$$
\begin{aligned}
\frac{\partial P}{\partial t}= & \left\{-\nabla \cdot F_{0}+\beta^{2} \int_{0}^{\infty} d \tau\left\langle\nabla \cdot F_{1}(t)\right.\right. \\
& \left.\left.\times \exp \left(-\tau \nabla \cdot F_{0}\right) \nabla \cdot F_{1}(t-\tau)\right\rangle \exp \left(\tau \nabla \cdot F_{0}\right)\right\} P .
\end{aligned}
$$

The operator $\exp \left(\tau \nabla \cdot F_{0}\right)$ in the above equation provides the solution to the equation

$$
\frac{\partial y(u, t)}{\partial t}=-\nabla \cdot F_{0} y(u, t)
$$

( $y$ signifies the unperturbed part of $\rho$ ), which can be found explicitly in terms of characteristic curves. The equation

$$
\dot{u}=F_{0}(u),
$$

for fixed $t$ determines a mapping from $u(\tau=0)$ to $u(\tau)$ i.e., $u \rightarrow u^{\tau}$ with the inverse $\left(u^{\tau}\right)^{-\tau}=u$. The solution of equation (A10) is

$$
y(u, t)=y\left(u^{-t}, 0\right)\left|\frac{d\left(u^{-t}\right)}{d(u)}\right|=\exp \left(-t \nabla \cdot F_{0}\right) y(u, 0),
$$

$\left|d\left(u^{-t}\right) / d(u)\right|$ being a Jacobian determinant. The effect of $\exp \left(-t \nabla \cdot F_{0}\right)$ on $y(u)$ is as follows:

$$
\exp \left(-t \nabla \cdot F_{0}\right) y(u, 0)=y\left(u^{-t}, 0\right)\left|\frac{d\left(u^{-t}\right)}{d(u)}\right| .
$$

The above simplification when put in equation A9 yields

$$
\begin{aligned}
\frac{\partial P(u, t)}{\partial t}= & \nabla \cdot\left\{-F_{0}+\beta^{2} \int_{0}^{\infty} d \tau\left|\frac{d\left(u^{-\tau}\right)}{d(u)}\right|\right. \\
& \times\left\langle F_{1}(u, t) \nabla_{-\tau} \cdot F_{1}\left(u^{-\tau}, t-\tau\right)\right\rangle \\
& \left.\times\left|\frac{d(u)}{d\left(u^{-\tau}\right)}\right|\right\} P(u, t) .
\end{aligned}
$$

where $\nabla_{-\tau}$ denotes differentiation with respect to $u^{-\tau}$. We put $\beta=1$ for the rest of the treatment. Identifying $u_{1}=x$ and $u_{2}=v$ we now write

$$
\begin{aligned}
& F_{01}=v, F_{11}=0, \\
& F_{02}=-\Gamma(x) v-V^{\prime}(x), \\
& F_{12}=h(x) f(t)+g_{1}(x) \epsilon(t)+g_{2}(x) \pi(t) .
\end{aligned}
$$

In this situation, equation n14 now reduces to

$$
\begin{aligned}
\frac{\partial P}{\partial t}= & -\frac{\partial}{\partial x}(v P)+\frac{\partial}{\partial v}\left\{\Gamma(x) v+V^{\prime}(x)\right\} P \\
& +\frac{\partial}{\partial v} \int_{0}^{\infty} d \tau\left\langle\left[ h(x) f(t)+g_{1}(x) \epsilon(t)+g_{2}(x) \pi(t)\right.\right. \\
& +\frac{\partial}{\partial v^{-\tau}}\left\{h\left(x^{-\tau}\right) f(t-\tau)+g_{1}\left(x^{-\tau}\right) \epsilon(t-\tau)\right. \\
& \left.\left.\left.\left.+g_{2}\left(x^{-\tau}\right) \pi(t-\tau)\right)\right\}\right]\right\rangle P,
\end{aligned}
$$

where we have used the fact that the Jacobian obeys the equation

$$
\frac{d}{d t} \log \left|\frac{d\left(x^{t}, v^{t}\right)}{d(x, v)}\right|=\frac{\partial v}{\partial x}+\frac{\partial}{\partial v}\left\{-\Gamma v+V^{\prime}(x)\right\}=-\Gamma,
$$

so that the Jacobian becomes $\exp (-\Gamma(x) t)$. Now neglecting the terms $O\left(\tau^{2}\right)$ we may have $x^{-\tau}=x-\tau v$ and $v^{-\tau}=v+\Gamma \tau v+\tau V(x)$. The above two equations yield

$$
\frac{\partial}{\partial v^{-\tau}}=(1-\Gamma \tau) \frac{\partial}{\partial v}+\tau \frac{\partial}{\partial x} .
$$

Taking this in to consideration of equation (A17), equation (A16) can be simplified in the following form:

$$
\begin{aligned}
\frac{\partial P}{\partial t}= & -\frac{\partial(v P)}{\partial x}+\frac{\partial}{\partial v}\left[\Gamma(x) v+V^{\prime}(x)\right] P \\
& +\frac{\partial^{2}}{\partial v^{2}}\left[k_{B} T \Gamma(x)+g^{2}(x)\right] P,
\end{aligned}
$$

where

$$
\begin{aligned}
g(x)= & \left\{D_{\epsilon} g_{1}^{2}(x)+2 \lambda \sqrt{D_{\epsilon} D_{\pi}} g_{1}(x) g_{2}(x)\right. \\
& \left.+D_{\pi} g_{2}^{2}(x)\right\}^{1 / 2} .
\end{aligned}
$$

Defining

$$
A(x)=k_{B} T \Gamma(x)+g^{2}(x),
$$

the above equation (A18) can be written as

$$
\begin{aligned}
\frac{\partial P}{\partial t}= & -v \frac{\partial P}{\partial x}+\left[\Gamma(x) v+V^{\prime}(x)\right] \frac{\partial P}{\partial v}+A(x) \frac{\partial^{2} P}{\partial v^{2}} \\
& +\Gamma(x) P,
\end{aligned}
$$

which is our required Fokker-Planck equation.

\section{APPENDIX B: CALCULATION OF THE ESCAPE RATE}

In this appendix we show the detailed calculation of the escape rate for nonequilibrium open system. The technique we adopt here resembles our previous approaches $32,33,38$ but makes the current paper self contained. 
Following Kramers, $\frac{1}{2}$ we make ansatz that the nonequilibrium steady state probability $P_{b}^{s t}(x, v)$ generating a non-vanishing diffusion current across the barrier is given by

$$
P_{b}^{s t}(x, v)=\exp \left(-\frac{v^{2}}{2 D_{b}}-\frac{V(x)}{D_{b}}\right) G(x, v),
$$

where $D_{b}=A_{b} / \Gamma\left(x_{b}\right)$. Inserting Eq. (B1) in (11), we obtain the equation for $G(x, v)$ using the steady state in the neighborhood of $x_{b}$

$$
-v \frac{\partial G}{\partial x}-\left[\omega_{b}^{2}\left(x-x_{b}\right)+\Gamma(x) v\right] \frac{\partial G}{\partial v}+A_{b} \frac{\partial^{2} G}{\partial v^{2}}=0 .
$$

At this point we set

$$
y=v+a\left(x-x_{b}\right),
$$

where $a$ is a constant to be determined. With the help of the transformation (B3), Eq. (B2) reduces to

$$
A_{b} \frac{d^{2} G}{d y^{2}}-\left[\omega_{b}^{2}\left(x-x_{b}\right)+\left\{\Gamma\left(x_{b}\right)+a\right\} v\right] \frac{d G}{d y}=0 .
$$

Now let

$$
\left[\omega_{b}^{2}\left(x-x_{b}\right)+\left\{\Gamma\left(x_{b}\right)+a\right\} v\right]=-\mu y,
$$

where $\mu$ is another constant. By virtue of the relation (B5), Eq.(B4) becomes

$$
\frac{d^{2} G}{d y^{2}}+\Lambda y \frac{d G}{d y}=0
$$

where $\Lambda=\mu / A_{b}$ with $A_{b}=k_{B} T \Gamma\left(x_{b}\right)+g^{2}\left(x_{b}\right)$. The constant $\mu$ and $a$ must satisfy the following equations simultaneously:

$$
-\mu a=\omega_{b}^{2} \text { and }-\mu=\Gamma\left(x_{b}\right)+a .
$$

This implies that the constant a must satisfy the following quadratic equation

$$
a^{2}+\Gamma\left(x_{b}\right) a-\omega_{b}^{2}=0,
$$

which allows the solution for $a$ as

$$
a_{ \pm}=\frac{1}{2}\left\{-\Gamma\left(x_{b}\right) \pm \sqrt{\Gamma^{2}\left(x_{b}\right)+4 \omega_{b}^{2}}\right\} .
$$

Thus, the general solution of (B6) is

$$
G(y)=G_{2} \int_{0}^{y} \exp \left(-\frac{\Lambda z^{2}}{2}\right) d z+G_{1},
$$

where $G_{1}$ and $G_{2}$ are two constants of integration. We look for a solution which vanishes for large $x$. This condition is satisfied if the integration in (B9) remains finite for $|y| \rightarrow+\infty$. This implies that $\Lambda>0$ so that only $a_{-}$ becomes relevant. Then the requirement $P_{b}(x, v) \rightarrow 0$ for $x \rightarrow+\infty$ yields

$$
G_{1}=G_{2} \sqrt{\frac{\pi}{2 \Lambda}}
$$

Thus we have

$$
G(y)=G_{2}\left[\sqrt{\frac{\pi}{2 \Lambda}}+\int_{0}^{y} \exp \left(-\frac{\Lambda z^{2}}{2}\right) d z\right],
$$

and correspondingly,

$$
\begin{aligned}
P_{b}^{s t}(x, v)= & G_{2}\left[\sqrt{\frac{\pi}{2 \Lambda}}+\int_{0}^{y} \exp \left(-\frac{\Lambda z^{2}}{2}\right) d z\right] \\
& \times \exp \left(-\frac{v^{2}}{2 D_{b}}-\frac{V(x)}{D_{b}}\right) .
\end{aligned}
$$

The current across the barrier associated with this steady state distribution is given by

$$
j=\int_{-\infty}^{+\infty} v P_{b}^{s t}\left(x \approx x_{b}, v\right) d v,
$$

which may be evaluated using (B12) and the linearized version of $V(x)$, namely $V(x) \approx E_{b}-\omega_{b}^{2}\left(x-x_{b}\right)^{2} / 2$ as

$$
j=G_{2} \sqrt{\frac{2 \pi}{\Lambda+D_{b}^{-1}}} D_{b} \exp \left(-\frac{E_{b}}{D_{b}}\right) .
$$

To determine the remaining constant $G_{2}$ we note that as $x \rightarrow-\infty$, the pre-exponential factor in (B12) reduces to $G_{2} \sqrt{2 \pi / \Lambda}$. We then obtain the reduced distribution function in $x$ as

$$
\tilde{P}_{b}^{s t}(x \rightarrow-\infty)=2 \pi G_{2} \sqrt{\frac{D_{b}}{\Lambda}} \exp \left(-\frac{V(x)}{D_{b}}\right),
$$

where we have used the definition for the reduced distribution $32,33,38$ as

$$
\tilde{P}(x)=\int_{+\infty}^{-\infty} P(x, v) d v .
$$

Similarly, we derive the reduced distribution in the left well around $x \approx x_{0}$ using Eq.(10) where the linearized potential is $V(x) \approx E_{0}+\omega_{0}^{2}\left(x-x_{0}\right)^{2} / 2$,

$$
\tilde{P}_{0}^{s t}(x)=\frac{1}{Z} \sqrt{2 \pi D_{0}} \exp \left(-\frac{\omega_{0}^{2}\left(x-x_{0}\right)^{2}}{2 D_{0}}\right),
$$

with the normalization constant given by $1 / Z=$ $\omega_{0} /\left(2 \pi D_{0}\right)$ The comparison of the distribution (B14) and (B15) near $x \approx x_{0}$, gives,

$$
G_{2}=\sqrt{\frac{\Lambda}{D_{b}}} \frac{\omega_{0}}{2 \pi \sqrt{2 \pi D_{0}}} .
$$

Hence, from (B13), the normalized current or the barrier crossing rate $k$, for moderate to large friction regime is given by

$$
k=\frac{\omega_{0}}{2 \pi} \frac{D_{b}}{\sqrt{D_{0}}} \sqrt{\frac{\Lambda}{1+\Lambda D_{b}}} \exp \left(-\frac{E}{D_{b}}\right),
$$

where $E=E_{b}-E_{0}$ is the potential barrier height. 
* Electronic address: jprc $8 @$ yahoo.com

$\dagger$ Electronic address: sudip chattopadhyay@rediffmail.com

$\ddagger$ Electronic address: skbanik@vt.edu; Present Address: Department of Biological Sciences, Virginia Polytechnic Institute and State University, Blacksburg, VA 24061-0406, USA.

1 H. A. Kramers, Physica 7, 284 (1940).

2 A. Simon and A. Libchaber, Phys. Rev. Lett. 68, 3375 (1992).

3 E.W.-G. Diau, J. L. Herek, Z. H. Kim, and A. H. Zewail, Science 279, 847 (1998).

4 L. I. McCann, M. I. Dykman, and B. Golding, Nature 402, 785 (1999)

5 P. Hänggi, P. Talkner, and M. Borkovec, Rev. Mod. Phys. 62, 251 (1990).

${ }^{6}$ V. I. Mel'nikov, Phys. Rep. 209, 1 (1991); H. Grabert, P. Schramm, and G. L. Ingold, Phys. Rep. 168, 115 (1998).

7 U. Weiss, Quantum Dissipative Systems (World Scientific, Singapore, 1999).

8 A. Nitzan, Chemical Dynamics in Condensed Phases (Oxford University Press, Oxford, 2006).

9 R. F. Grote and J. T. Hynes, J. Chem. Phys. 73, 2715 (1980).

10 E. Pollak, J. Chem. Phys. 85, 865 (1986).

11 K. Okamura and Y. Tanimura, Phys. Rev. E 56, 2747 (1997); T. Kato and Y. Tanimura, J. Chem. Phys. 120, 260 (2004).

12 P. Resibois and M. dc Leener, Chemical Kinetic Theory of Fluids (Wiley-Interscience, New York, 1977).

13 J. R. Chaudhuri, G. Gangopadhyay, and D. S. Ray, J. Chem. Phys. 109, 5565 (1998).

14 R. Hernandez, J. Chem. Phys. 111, 7701 (1999); R. Hernandez and F. L. Somer, J. Phys. Chem. B 103, 1064 (1999).

15 E. Hershkovits and R. Hernandez, J. Chem. Phys. 122, 014509 (2005).

16 J. M. Moix, T. D. Shepherd, and R. Hernandez, J. Phys. Chem. B 108, 19476 (2004); J. M. Moix and R. Hernandez, J. Chem. Phys. 122, 114111 (2005).

17 A. V. Popov and R. Hernandez, J. Chem. Phys. 126, 244506 (2007).

18 H. W. Hsia, N. Fang, and X. Lee, Phys. Lett. A 215, 326 (1996); A. N. Drozdov and S. C. Tucker, J. Phys. Chem. B 105, 6675 (2001).

19 J. R. Chaudhuri, D. Barik, and S.K. Banik, J. Phys. A 40, 14715 (2007).

20 J. R. Chaudhuri, S. Chattopadhyay, and S.K. Banik, J. Chem. Phys. 127, 224508 (2007).

21 J. R. Chaudhuri, D. Barik, and S. K. Banik, Phys. Rev. E 74, 061119 (2006).

22 E. Pollak and P. Talkner, Chaos 15, 026116 (2005) and references therein.

23 E. Pollak and A. M. Berezhkovskii, J. Chem. Phys. 99, 1344 (1993)

24 Y. M. Blanter and M. Büttiker, Phys. Rev. Lett. 81, 4040 (1998); Phys. Rep. 336, 1 (2000); R. Krishnan, M. C. Mahato, and A. M. Jayannavar, Phys. Rev. E 70, 021102 (2004).

25 M. O. Magnasco, Phys. Rev. Lett. 71, 1477 (1993).

26 P. Reimann, M. Grifoni, and P. Hänggi, Phys. Rev. Lett. 79, 10 (1997); P. Reimann, Phys. Rep. 361, 57 (2002).
27 F. Marchesoni, Chem. Phys. Lett. 110, 20 (1984); A. V. Barzykin and K. Seki, Europhys. Lett. 40, 117 (1997); Q. Long, L. Cao, Da-jin Wu, and Zai-guang Li, Phys. Lett. A 231, 339 (1997); O. V. Gerashchenko, S. L. Ginzburg, and M. A. Pustovoit, JETP Lett. 67, 997 (1998).

28 R. L. Stratonovich, Topics in the Theory of Random Noise (Gordon and Breach, London, 1967).

29 R. Kubo, M. Toda, N. Hashitsume, and N. Saito, Statistical Physics II: Nonequilibrium Statistical Mechanics (Springer, Berlin, 1995).

30 W. Horsthemke and R. Lefever, Noise-induced Transitions (Springer, Berlin, 1994).

31 K. Huang, Lectures on Statistical Physics and Protein Folding (World Scientific, Singapore, 2005).

32 J. R. Chaudhuri, S. K. Banik, B. C. Bag, and D. S. Ray, Phys. Rev. E. 63, 061111 (2001).

33 S. K. Banik, J. Ray Chaudhuri, and D. S. Ray, J. Chem. Phys. 112, 8330 (2000).

34 J. R. Chaudhuri, D. Barik, and S. K. Banik, Phys. Rev. E 73, 051101 (2006).

35 J. Mencia Bravo, R. M. Velasco, and J. M. Sancho, J. Math. Phys. 30, 2023 (1989).

${ }^{36}$ K. Lindenberg and B. J. West, The Nonequilibrium Statistical Mechanics of Open and Closed Systems (VCH, New York, 1990).

37 M. M. Millonas and C. Ray, Phys. Rev. Lett. 75, 1110 (1995).

38 J. R. Chaudhuri, S. Chattopadhyay, and S. K. Banik, Phys. Rev. E 76, 021125 (2007).

39 I.I. Fedchenia, J. Stat. Phys. 52, 1005 (1988).

40 A. Fulinski and T. Telejko, Phys. Letts. A 152, 11 (1991).

41 A. J. R. Madureira, P. Hänggi, and H. S. Wio, Phys. Lett. A 217, 248 (1994).

42 D. Mei, C. Xie, and L. Zhang, Phys. Rev. E 68, 051102 (2003).

43 S. Zhu, Phys. Rev. A 47, 2405 (1993).

44 Y. Jia and J. R. Li, Phys. Rev. E 53, 5786 (1996).

45 V. Berdichevsky and M. Gitterman, Phys. Rev. E 60, 1494 (1999).

46 B. C. Bag, S. K. Banik, and D. S. Ray, Phys. Rev. E 64, 026110 (2001).

47 C. J. Tessone, H. S. Wio, and P. Hänggi, Phys. Rev. E 62, 4623 (2000)

48 J. Li, J. Łuczka, and P. Hänggi Phys. Rev. E 64, 011113 (2001).

49 B. Q. Ai, X. J. Wang, G. T. Liu, and L. G. Liu, Phys. Rev. E 67, 022903 (2003).

${ }^{50}$ P. K. Ghosh, B. C. Bag, and D. S. Ray, Phys. Rev. E 75, 032101 (2007); J. Chem. Phys. 127, 044510 (2007).

51 P. Jung, Phys. Rep. 234, 175 (1993); C. Xie, D. Mei, L. Cao, and Da-jin Wu, Eur. Phys. J. B 33, 83 (2003); P. Majee and B. C. Bag, J. Phys. A 37, 3353 (2004).

52 J. Garcia-Ojalvo and J. M. Sancho, Noise in spatially extended system (Springer-Verlag, New York, 1999).

53 J. M. Sancho, M. San Miguel, S. L. Katz, and J. D. Gunton, Phys. Rev. A 26, 1589 (1982).

${ }^{54}$ C. Mahanta and T.G. Venkatesh, Phys. Rev. E 58, 4141 (1998); D. Barik, B. C. Bag, and D. S. Ray, J. Chem. Phys. 119, 12973 (2003).

55 N. G. van Kampen, Phys. Rep. 24, 1710 (1976). 